Quim. Nova, Vol. 34, No. 8, 1358-1364, 2011

\title{
ANÁLISE DE PINTURAS RUPESTRES DO ABRIGO DO JANELÃO (MINAS GERAIS) POR MICROSCOPIA RAMAN
}

\author{
Dalva Lúcia A. de Faria* e Francisco N. Lopes \\ Departamento de Química Fundamental, Instituto de Química, Universidade de São Paulo, CP 26077, 05513-970 São Paulo - SP, \\ Brasil \\ Luiz Antônio Cruz Souza e Helena David de Oliveira Castello Branco ${ }^{\dagger}$ \\ Centro de Conservação e Restauração de Bens Culturais Móveis, Escola de Belas Artes, Universidade Federal de Minas Gerais, \\ Av. Antonio Carlos, 6627, 31270-901 Belo Horizonte - MG, Brasil
}

Recebido em 22/11/10; aceito em 25/3/11; publicado na web em 10/6/11

\begin{abstract}
ROCK ART FROM ABRIGO DO JANELÃO (MINAS GERAIS) INVESTIGATED BY RAMAN MICROSCOPY. Rock art paintings from Abrigo do Janelão (Minas Gerais, Brazil) were non-destructively investigated by Raman Microscopy, aiming at the identification of materials used, their interaction and degradation. This technique is particularly tailored for heterogeneous samples and allows unequivocal identification of the substances present in the investigated sample.

Pigments, were identified together with products of microbiological degradation; no binders were detected. White pigment was identified as calcite $\left(\mathrm{CaCO}_{3}\right)$, whereas charcoal was used as black, goethite $(\alpha-\mathrm{FeOOH})$ as yellow and hematite $\left(\alpha-\mathrm{Fe}_{2} \mathrm{O}_{3}\right)$ as red. Whewellite $\left(\mathrm{CaC}_{2} \mathrm{O}_{4} \cdot \mathrm{H}_{2} \mathrm{O}\right)$ and weddelite $\left(\mathrm{CaC}_{2} \mathrm{O}_{4} \cdot 2 \mathrm{H}_{2} \mathrm{O}\right)$ were detected and their origin was assigned to degradation products from microbiological activity.
\end{abstract}

Keywords: rock art; pigments; Raman.

\section{INTRODUÇÃO}

O estudo de problemas ligados a bens culturais móveis e imóveis vem ganhando impulso em todo o mundo, inclusive no Brasil, devido a uma série de fatores que englobam a necessidade de preservar objetos cuja importância se estende além dos valores comerciais e estéticos, uma vez que geralmente estão ligados à identidade de povos e etnias. Nesse contexto, a arte rupestre é um tópico dos mais atrativos, por estar ligada aos primórdios do desenvolvimento de sociedades, fazendo com que uma maior compreensão das técnicas e dos recursos empregados possa ampliar o entendimento de aspectos antropológicos e históricos desses povos.

As pesquisas em arte rupestre no Brasil intensificaram-se a partir do início da década de 1970, com os estudos realizados no Piauí, que culminaram na criação do Parque Nacional Serra da Capivara (1979). ${ }^{1}$ Nas três últimas décadas, novas descobertas impulsionaram os estudos e ampliou-se o número de centros de pesquisa interessados na questão da ocupação do continente americano, o que é evidenciado pela produção de dissertações, teses e publicações nacionais e internacionais. ${ }^{2}$ Hoje, as investigações envolvem vários grupos de pesquisa consolidados, dentre os quais destacam-se os trabalhos que vêm sendo feitos pela equipe de pesquisadores da FUMDHAM (Fundação Museu do Homem Americano) ${ }^{3}$ e da UFMG, ${ }^{4}$ além de projetos desenvolvidos pelo Museu de Arqueologia e Etnologia da USP. ${ }^{5}$

A utilização de técnicas físico-químicas na caracterização das tintas ou de processos de degradação encontradas nas representações pictóricas tem um papel fundamental nas investigações pela precisão das informaç̃oes obtidas. No Brasil, pesquisas envolvendo os pigmentos utilizados e sua interação com o ambiente ainda são incipientes. ${ }^{4}$

\footnotetext{
${ }^{\dagger}$ In memoriam

*e-mail: dlafaria@iq.usp.br
}

A literatura revela que as técnicas não destrutivas que mais vêm sendo empregadas no estudo de problemas ligados ao patrimônio histórico, artístico e cultural são a microscopia Raman e a fluorescência de raios X (XRF). Entre elas, a microscopia Raman é aquela que fornece a maior quantidade de informações sobre o objeto analisado, além de ser uma técnica não destrutiva, que não demanda qualquer tipo de manipulação da amostra ou do objeto. Por esse motivo, essa técnica vem dando significativa contribuição por proporcionar amplas informações em termos de composição química, tanto para compostos orgânicos como inorgânicos. ${ }^{6}$

Especificamente no que diz respeito à arte rupestre, a microscopia Raman vem sendo usada com muito sucesso. Trabalhos publicados por Smith et al.,${ }^{7}$ Edwards et al.,${ }^{8}$ Russ et al. ${ }^{9}$ e Hernanz et al. ${ }^{10}$ objetivavam a identificação de substâncias, especificamente pigmentos, empregadas em pinturas rupestres, a caracterização de metodologias eventualmente envolvidas na preparação e aplicação das tintas, assim como a detecção de degradações de origem microbiológica sobre as pinturas. Mais recentemente, amostras de pigmentos coletadas em sítio arqueológico na Patagônia Argentina foram caracterizadas através do uso combinado de técnicas de espectroscopia vibracional e de métodos cromatográficos ${ }^{11}$ e, em outro trabalho, investigou-se a conversão de goetita a hematita através de aquecimento, procurando esclarecer aspectos ligados à manipulação de pigmentos por culturas pré-históricas. ${ }^{12}$

O sucesso obtido na investigação de problemas ligados à pintura rupestre suscitou o interesse em ampliar o conhecimento acerca dos sítios arqueológicos brasileiros. Este trabalho trata de análises feitas para pigmentos coletados no Vale do Peruaçu, município de Januária, norte de Minas Gerais, o qual contém uma vasta gama de inscrições rupestres sobre rocha, tornando-o um local privilegiado para estudos. A área vem sendo estudada desde a década de 1970 pelo Setor de Arqueologia do Museu de História Natural e Jardim Botânico da UFMG, coordenado por André Prous. ${ }^{4}$ 
A diversidade caracteriza as pinturas rupestres presentes nesse sítio, as quais representam figuras geométricas, antropomórficas e zoomórficas, além de alguns fitomorfos. As cores detectadas a olho nu são o preto, o branco, o vermelho (vários tons), alaranjado, amarelo e até mesmo a cor verde que, na verdade, se verificou posteriormente se tratar de mistura dos pigmentos preto e amarelo. Os fragmentos analisados foram retirados de representações de diversos tipos e tamanhos, segundo protocolos que garantissem um mínimo de dano às pinturas. ${ }^{13}$

O estudo de pigmentos questiona principalmente sua composição química, o uso de cargas e/ou aglutinantes, a formação de depósitos posteriores sobre a superfície desses pigmentos e alterações provocadas por intemperismos (umidade, temperatura etc.), além de produtos de degradação promovida por micro-organismos. Do ponto de vista da conservação, o estudo sistemático do local justifica-se também pela aceleração da ação direta do homem como decorrência de turismo depredatório, exploração madeireira, queimadas e ocupação rural desordenada.

Este trabalho objetivou, portanto, contribuir para a identificação dos pigmentos e de eventuais cargas ou aglutinantes empregados, além de seus processos de degradação. Este último aspecto é extremamente importante para a definição de estratégias de conservação preventiva desses sítios arqueológicos.

\section{PARTE EXPERIMENTAL}

As amostras contendo pigmentos rupestres foram coletadas por Helena Castello Branco, como parte integrante de sua dissertação de mestrado orientada por Luiz Antônio de Souza, do Centro de Conservação e Restauração (Cecor) da Escola de Belas Artes da UFMG. ${ }^{13} \mathrm{O}$ sítio escolhido foi o Abrigo Norte do Janelão, localizado no Parque Nacional Cavernas do Peruaçu, em Minas Gerais. Foram coletadas 40 amostras, dentre as quais 32 do Abrigo do Janelão, que é um grande abrigo sob rocha calcária, com mais de $70 \mathrm{~m}$ de comprimento e entre 80 e $100 \mathrm{~m}$ de altura. Está situado na margem esquerda do rio Peruaçu, a cerca de $100 \mathrm{~m}$ do leito, na entrada da Gruta do Janelão $\left(15^{\circ} 06.84\right.$ ' S / 44 $\left.{ }^{\circ} 14.56^{\prime} \mathrm{W}\right) .{ }^{13}$ Das 32 amostras coletadas do Abrigo do Janelão, foram selecionadas 14 para análise por microscopia Raman. Tais amostras são constituídas de minúsculos fragmentos (a partir de 10 $\mu \mathrm{m}^{3}$ ) ou de pequenos grânulos de ca. $1 \mathrm{~mm}^{2}$, de pigmentos nas cores preta, branca, amarela e vermelha, retirados de localidades diferentes do sítio e que foram obtidas diretamente dos painéis rochosos com a pintura e as degradações presentes, para identificação dos pigmentos e do material degradado.

As amostras foram analisadas diretamente sobre lâminas de microscópio, sem tratamento prévio e apresentaram-se bastante heterogêneas, constituindo-se basicamente do material pigmentado, da rocha de suporte e de deteriorações que poderiam ser devidas a intempéries e/ou à ação de micro-organismos e insetos.

Amostras de oxalato de cálcio mono-hidratado $\left(\mathrm{CaC}_{2} \mathrm{O}_{4} \cdot \mathrm{H}_{2} \mathrm{O}\right.$, wewelita) e de oxalato de cálcio di-hidratado $\left(\mathrm{CaC}_{2} \mathrm{O}_{4} \cdot 2 \mathrm{H}_{2} \mathrm{O}\right.$, wedelita), foram preparadas segundo procedimento descrito na literatura. ${ }^{14,15}$
Wewelita foi obtida pela precipitação de ácido oxálico $\left(\mathrm{C}_{2} \mathrm{O}_{2} \mathrm{H}_{2} \cdot 2 \mathrm{H}_{2} \mathrm{O}\right)$ $0,1 \mathrm{~mol} / \mathrm{L}$ com solução $0,1 \mathrm{~mol} / \mathrm{L}$ de $\mathrm{CaCl}_{2}$ à temperatura ambiente. Wedelita é a forma mais instável, obtida de soluções mais diluídas e em temperaturas menores, condições nas quais a coprecipitação de wewelita é evitada. Para isso usou-se solução $0,02 \mathrm{~mol} / \mathrm{L}$ de ácido oxálico, alcalinizada até $\mathrm{pH} 8,0$ e adicionada gota a gota à solução de $\mathrm{CaCl}_{2}$ $0,02 \mathrm{~mol} / \mathrm{L}$, sob agitação vigorosa em temperaturas entre 5 e $10^{\circ} \mathrm{C}$.

Os espectros Raman foram obtidos em microscópio Raman Renishaw system 3000, dotado de detector CCD (Wright, $600 \mathrm{x}$ 400 pixels) refrigerado termoeletricamente $\left(-20^{\circ} \mathrm{C}\right)$ e acoplado a um microscópio metalúrgico Olympus (BH2-UMA). As amostras foram estudadas sem qualquer tipo de preparação ou manipulação especial. Todos os espectros foram analisados com uso de radiação em 632,8 nm (laser de He-Ne, Spectra Physics, mod. 127) e a potência na amostra foi tipicamente $0,5 \mathrm{~mW}$.

Os espectros de absorção no IR foram obtidos em equipamento FTIR Bomem MB100, usando entre 64 e 512 acumulações e resolução de $4 \mathrm{~cm}^{-1}$. Para aquisição de espectros de ATR foi utilizado acessório de ATR de reflexão simples Silver Gate ${ }^{\mathrm{TM}}$ (Specac), dotado de cristal de Ge.

\section{RESULTADOS E DISCUSSÃO}

As análises foram realizadas buscando não só a caracterização dos pigmentos empregados nas representações pictóricas, como também outros componentes da tinta, como aglutinantes por exemplo, ou então algum vestígio da metodologia de sua preparação ou aplicação. Outros aspectos de interesse são também proporcionados por depósitos de contaminantes antropogênicos, como fuligem por exemplo, além de agentes microbiológicos. Os espectros mostrados a seguir (Figuras 1 a 4) são representativos das amostras analisadas e descritas na Tabela 1. A Tabela 2 apresenta as bandas características dos pigmentos encontrados em cada amostra.

\section{Pigmentos pretos}

Os espectros Raman confirmaram a presença de carbono amorfo em três amostras, 1114T, 1125T e 1130T. Na Figura1, é possível notar o perfil alargado típico do material com baixa organização estrutural e que é expresso pela grande intensidade da banda em torno de 1360 $\mathrm{cm}^{-1}$, chamada de banda $\mathrm{D}$, característica da vibração de estiramento da ligação C-C com baixa ordem de ligação. ${ }^{16} \mathrm{Em} 1580 \mathrm{~cm}^{-1}$, esse desordenamento do material é também expresso pelo alargamento da banda que tem contribuição majoritária associada à vibração $\mathrm{C}-\mathrm{C}$ com maior ordem de ligação (banda G). ${ }^{16}$

De acordo com a ref.13, na qual as técnicas SEM, FTIR, XRD e PLM foram empregadas na caracterização das mesmas amostras, há duas delas que apresentaram altos valores de manganês nas análises por SEM, o que poderia indicar o uso de minérios desse metal como pigmentos pretos ou marrons. Neste trabalho, porém, essa possi-

Tabela 1. Planilha descrevendo as amostras provenientes do Abrigo Norte do Janelão que foram analisadas por microscopia Raman (adaptada da ref. 14)

\begin{tabular}{lccc}
\hline Amostra & Descrição/proveniência & Amostra & Descrição/proveniência \\
\hline $1110 \mathrm{~T}$ & Superfície branca & $1128 \mathrm{~T}$ & Fragmento de pintura vermelha com camada branca por cima \\
$1113 \mathrm{~T}$ & Escama branca, dura & $1130 \mathrm{~T}$ & Fragmento de pintura "verde" que contorna figura amarela \\
$1114 \mathrm{~T}$ & Fragmento de camada pictórica preta & $1131 \mathrm{~T}$ & Fragmento de pintura amarela, com escamação e pigmentos pretos \\
$1120 \mathrm{~T}$ & Fragmento de material branco por baixo de camada pictórica & $1132 \mathrm{~T}$ & Fragmento de pintura branca que contorna figura vermelha \\
$1121 \mathrm{~T}$ & Fragmento de camada pictórica com presença de material branco & $1133 \mathrm{~T}$ & Fragmento de figura vermelha com material branco e escamação \\
$1124 \mathrm{~T}$ & Fragmento de figura branca & 1134T & Fragmento de figura vermelha com perdas e material branco \\
$1125 \mathrm{~T}$ & Fragmento de figura preta, traços finos & $1135 \mathrm{~T}$ & Material branco coletado de figura vermelha
\end{tabular}


Tabela 2. Resumo das substâncias identificadas das amostras do Abrigo Norte do Vale do Peruaçu e respectivas frequências de vibração Raman

\begin{tabular}{|c|c|c|c|}
\hline Amostras & $\begin{array}{l}\text { Substâncias } \\
\text { identificadas }\end{array}$ & Composição & $\begin{array}{c}\text { Posição/cm }{ }^{-1} \mathrm{e} \\
\text { intensidade relativa* }\end{array}$ \\
\hline $\begin{array}{l}1114 \mathrm{~T}, 1125 \mathrm{~T}, \\
1130 \mathrm{~T}\end{array}$ & carvão & carbono amorfo & $\begin{array}{l}1360 \mathrm{~s} \\
1580 \mathrm{~s}\end{array}$ \\
\hline $\begin{array}{l}1110 \mathrm{~T}, 1113 \mathrm{~T} \\
1124 \mathrm{~T}, 1132 \mathrm{~T}\end{array}$ & calcita & $\begin{array}{l}\text { carbonato de } \\
\text { cálcio, } \mathrm{CaCO}_{3}\end{array}$ & $\begin{array}{c}156 \mathrm{~m} \\
283 \mathrm{~m} \\
713 \mathrm{~m} \\
1088 \mathrm{~m}\end{array}$ \\
\hline $\begin{array}{l}\text { 1128T, 1133T, } \\
1134 \mathrm{~T}\end{array}$ & hematita & $\begin{array}{c}\text { óxido de ferro, } \\
\alpha-\mathrm{Fe}_{2} \mathrm{O}_{3}\end{array}$ & $\begin{array}{c}225 \mathrm{~s} \\
243 \mathrm{w} \\
290 \mathrm{~s} \\
299 \mathrm{sh} \\
408 \mathrm{~m} \\
499 \mathrm{w} \\
610 \mathrm{~m} \\
660 \mathrm{~s} \\
1320 \mathrm{~s}\end{array}$ \\
\hline $1130 \mathrm{~T}, 1131 \mathrm{~T}$ & goetita & $\begin{array}{l}\text { oxi-hidróxido de } \\
\text { ferro, } \alpha \text {-FeOOH }\end{array}$ & $\begin{array}{l}245 \mathrm{w} \\
298 \mathrm{~m} \\
383 \mathrm{~s} \\
477 \mathrm{w} \\
549 \mathrm{~m}\end{array}$ \\
\hline $\begin{array}{l}1110 \mathrm{~T}, 1120 \mathrm{~T}, \\
1121 \mathrm{~T}, 1128 \mathrm{~T}, \\
1133 \mathrm{~T}, 1135 \mathrm{~T}\end{array}$ & wewelita & $\begin{array}{l}\text { oxalato de cálcio, } \\
\text { mono-hidratado, } \\
\mathrm{CaC}_{2} \mathrm{O}_{4} \cdot \mathrm{H}_{2} \mathrm{O}\end{array}$ & $\begin{array}{l}506 \mathrm{~m} \\
906 \mathrm{~m} \\
1465 \mathrm{~s} \\
1492 \mathrm{~s}\end{array}$ \\
\hline $\begin{array}{l}1114 \mathrm{~T}, 1130 \mathrm{~T}, \\
1134 \mathrm{~T}\end{array}$ & wedelita & $\begin{array}{c}\mathrm{CaC}_{2} \mathrm{O}_{4} \cdot 2 \mathrm{H}_{2} \mathrm{O} \text { e } \\
\text { outros metabólitos }\end{array}$ & $\begin{array}{c}504 \mathrm{~m} \\
909 \mathrm{~m} \\
1475 \mathrm{~s} \\
1135 \mathrm{vw} \\
1147 \mathrm{vw} \\
1337 \mathrm{w} \\
1522 \mathrm{vw} \\
1629 \mathrm{w}\end{array}$ \\
\hline
\end{tabular}

$* \mathrm{vw}=$ muito fraca, $\mathrm{w}=$ fraca, $\mathrm{m}=$ média, $\mathrm{s}=$ forte, $\mathrm{sh}=$ ombro

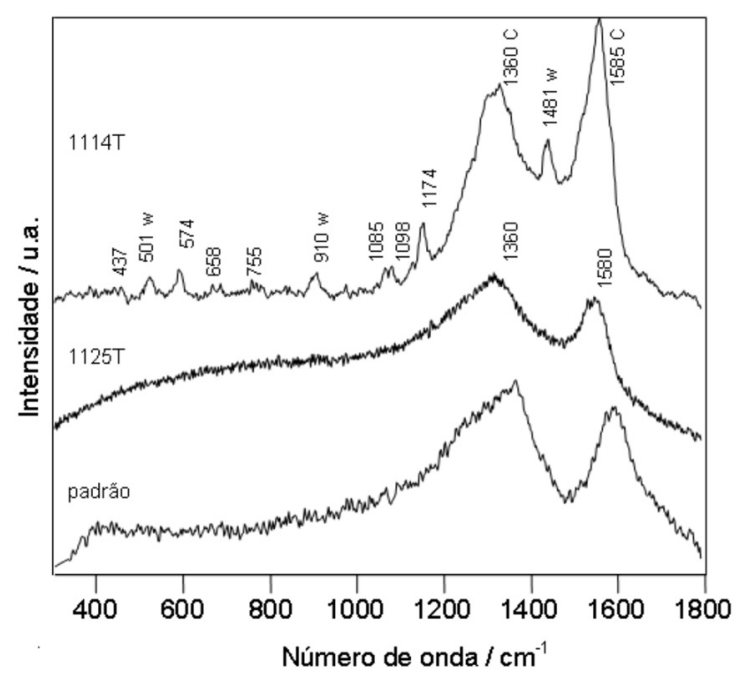

Figura 1. Espectros Raman $(632,8 \mathrm{~nm})$ de pigmentos pretos nas amostras 1114 T e 1125T, com as bandas de carbono amorfo assinaladas (C). A amostra 1114 apresenta bandas de wedelita (w). Um espectro de carbono amorfo (padrão) foi incluído para comparação bilidade não se confirmou. É importante ressaltar, entretanto, que há bastante divergência na literatura acerca de espectros Raman de óxidos de manganês, devido à sua relativa instabilidade. ${ }^{17}$ Além disso, como são fracos espalhadores de radiação suas bandas Raman são facilmente mascaradas por ruídos de fundo ou por outras substâncias, como óxidos de ferro que apresentam bandas na mesma região, entre 500 e $700 \mathrm{~cm}^{-1}$. Na amostra $1114 \mathrm{~T}$ outras bandas são visíveis e serão discutidas oportunamente.

Carvão também foi detectado em amostras de coloração amarela (1130T e 1131T). A olho nu essas regiões têm aparência esverdeada, porém, ao microscópio óptico, verifica-se que resultam da mistura de pigmento preto e amarelo, fato confirmado pela microscopia Raman que detectou a presença de carvão e goetita $(\alpha-\mathrm{FeOOH}$, espectro será discutido abaixo) nas regiões de cor esverdeada. Bandas em 909 e $1479 \mathrm{~cm}^{-1}$, atribuídas à wedelita, são sinais da ação de microorganismos e serão discutidas oportunamente.

\section{Pigmentos vermelhos}

Em todos os pontos das amostras analisadas, o perfil do espectro de hematita se repetiu com maior ou menor qualidade para as amostras 1128T, 1133T e 1134T. Os espectros, mostrados na Figura 2 confirmam a mesma identificação já obtida anteriormente por testes microquímicos e por PLM, ${ }^{13}$ ambos métodos destrutivos ou que exigem manipulação da amostra. As bandas da hematita nesses espectros $\left(293,411,502,614\right.$ e $\left.1320 \mathrm{~cm}^{-1}\right)$ são alargadas, indicando um certo grau de desordenamento. ${ }^{13} \mathrm{~A}$ banda em $1320 \mathrm{~cm}^{-1}$, atribuída inicialmente ao espalhamento de magnon da estrutura magnética do cristal $^{18}$ é atualmente descrita como um sobretom associado a um fónon óptico longitudinal (LO) em torno de $660 \mathrm{~cm}^{-1}$, proibido por regras de seleção. ${ }^{19}$ Esta última banda poderia ser sensível à desorganização estrutural do cristal a qual, por sua vez, poderia decorrer de intemperismos, sofridos pelo pigmento ao longo do tempo. Novamente, outras bandas são observadas no espectro da amostra 1134T (909 e $1480 \mathrm{~cm}^{-1}$ ) e na amostra $1128 \mathrm{~T}$ (1464 e $1492 \mathrm{~cm}^{-1}$ ), que não estão relacionadas a vibrações desses pigmentos e serão discutidas adiante.

\section{Pigmentos brancos}

As amostras 1124T, 1132T e $1197 \mathrm{~T}$ indicaram a presença de calcita $\left(\mathrm{CaCO}_{3}\right)$. São bem evidentes a banda característica de estiramento simétrico de $\mathrm{C}-\mathrm{O}$ do grupo $\left(\mathrm{CO}_{3}{ }^{2-}\right)$ em $1086 \mathrm{~cm}^{-1} \mathrm{e}$ a banda em $281 \mathrm{~cm}^{-1}$ (deformação angular fora do plano das ligações C-O); a banda em $712 \mathrm{~cm}^{-1}$ relativa à deformação angular no plano do íon $\mathrm{CO}_{3}{ }^{2-}$ também é característica desse composto. ${ }^{20}$ Aqui é importante esclarecer que o carbonato de cálcio aparece na natureza em três formas cristalinas: calcita, aragonita e vaterita, as quais podem ser diferenciadas por espectroscopia Raman porque, apesar de terem a mesma composição, possuem arranjos cristalinos distintos. Esse é um outro aspecto que diferencia a espectroscopia Raman de várias outras técnicas, usualmente empregadas em estudos de bens culturais.

As demais bandas em 502, 895, 1464 e $1496 \mathrm{~cm}^{-1}$ são devidas a oxalato de cálcio na forma mono-hidratada (Figura 3). Esse composto pode ser considerado um produto de degradação, porque resulta da reação do ácido oxálico, produzido pelo metabolismo de líquens, com o carbonato de cálcio do substrato rochoso. A amostra $1132 \mathrm{~T}$ provém de traços finos que contornam uma figura vermelha e nela não foi encontrada caolinita (silicato de alumínio hidratado, $\mathrm{Al}_{2} \mathrm{O}_{3} \cdot 2 \mathrm{SiO}_{2} \cdot 2 \mathrm{H}_{2} \mathrm{O}$ ), cuja presença foi sugerida anteriormente. ${ }^{13} \mathrm{O}$ espectro Raman de aluminossilicatos apresenta bandas de estiramento do grupo $\mathrm{OH}$ (acima de $3500 \mathrm{~cm}^{-1}$ ), de vibrações dos tetraedros de sílica (entre 100 e $600 \mathrm{~cm}^{-1}$ ) e dos octaedros de alumina (entre 300 e $1000 \mathrm{~cm}^{-1}$ ). ${ }^{21}$ Dessas vibrações, a mais intensa 


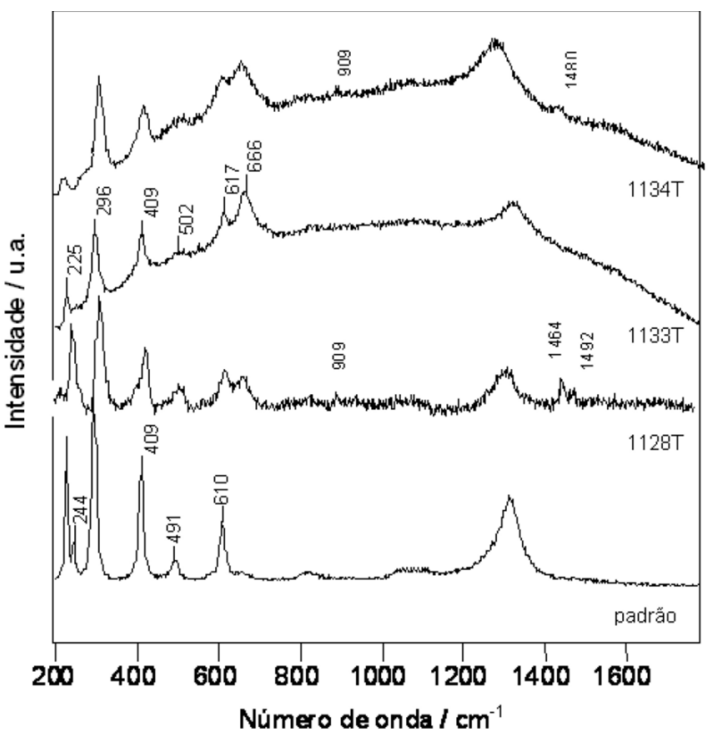

Figura 2. Espectros Raman $(632,8 \mathrm{~nm})$ de pigmento vermelho nas amostras 1134T, $1133 T$ e 1128T. Um espectro de hematita (padrão, $\alpha-F e_{2} O_{3}$ ) foi incluído para comparação

na região considerada nos espectros aqui reportados aparece em ca. $140 \mathrm{~cm}^{-1}$, a qual claramente não é observada na Figura 3 e nem em outros espectros registrados para a mesma amostra e que não são mostrados na figura.

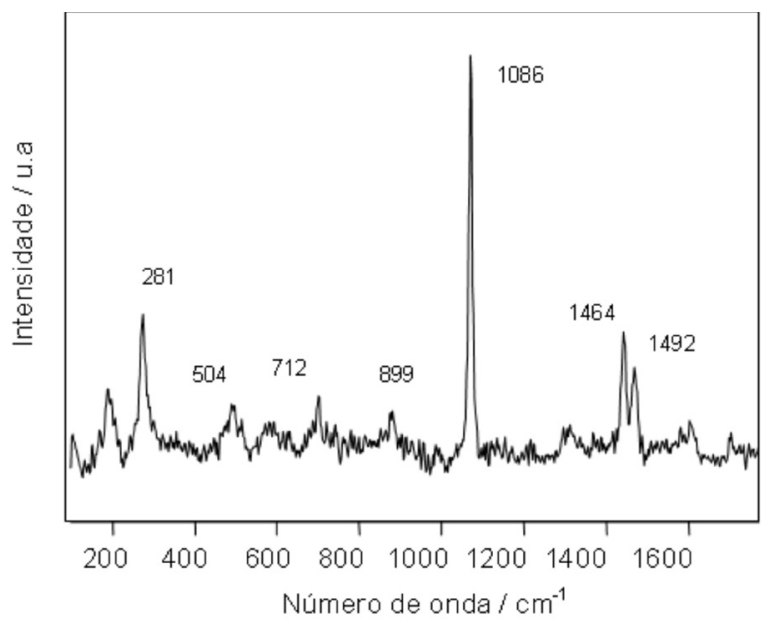

Figura 3. Espectro Raman (632,8 nm) de pigmento branco na amostra 1124T, com bandas em 281, 712 e $1086 \mathrm{~cm}^{-1}$ referentes à calcita $\left(\mathrm{CaCO}_{3}\right)$; as bandas 504,899 e 1464 e $1492 \mathrm{~cm}^{-1}$ são características de wewelita $\left(\mathrm{CaC}_{2} \mathrm{O}_{4} \cdot \mathrm{H}_{2} \mathrm{O}\right)$

\section{Pigmentos amarelos}

Goetita (oxi-hidróxido de ferro, $\alpha-\mathrm{FeOOH}$ ) foi identificado em duas amostras, $1130 \mathrm{~T}$ e 1131T. As bandas em baixa frequência, relativas às vibrações do octaedro $\mathrm{Fe}-\mathrm{O}$, aparecem em 245, 298, 383, 477,549 e $686 \mathrm{~cm}^{-1}$ (Figura 4), concordantes com a literatura. ${ }^{22} \mathrm{Em}$ alguns pontos da amostra $1130 \mathrm{~T}$ descrita como sendo proveniente de um contorno verde de uma figura geométrica amarela detectou-se mistura de goetita com carvão, como discutido acima; wewelita foi também detectada nessa amostra. Já nos espectros Raman da amostra $1131 \mathrm{~T}$, além de carvão e goetita foi também detectada wedelita $\left(\mathrm{CaC}_{2} \mathrm{O}_{4} \cdot 2 \mathrm{H}_{2} \mathrm{O}\right)$.

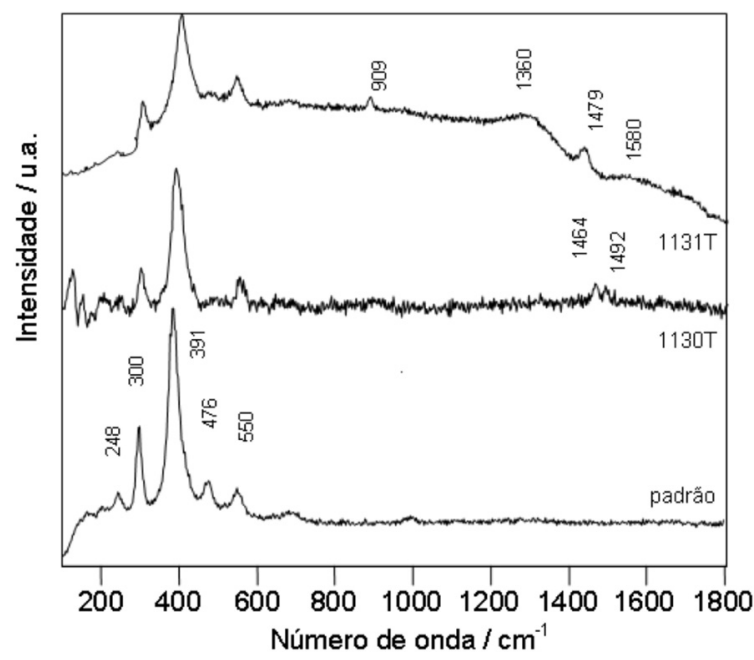

Figura 4. Espectros Raman $(632,8 \mathrm{~nm})$ de pigmento amarelo (goetita, $\alpha-\mathrm{FeOOH}$ ) nas amostras 1130T e 1131T. Na amostra 1131T, além do pigmento são observadas bandas da wewelita (909 e $\left.1479 \mathrm{~cm}^{-1}\right)$ e de carvão (1360 e $1580 \mathrm{~cm}^{-1}$ ). Na amostra 1130T, bandas em 909, $1464 \mathrm{e} 1492 \mathrm{~cm}^{-1}$ indicam a presença de wedelita. O espectro de goetita foi incluído para comparação

\section{Aglutinantes e cargas}

Não foram encontradas evidências da presença de aglutinantes orgânicos ou fixadores, como gordura animal, cola de peixe, extrato vegetal, sangue, ovo etc., que geralmente são detectáveis entre 800 e $1700 \mathrm{~cm}^{-1}$. A presença de aglutinantes poderia ser inferida, por exemplo, por bandas em 1670 e $1245 \mathrm{~cm}^{-1}$, relativas a vibrações $v(C=O)$ de amida I e $\delta(\mathrm{NH})$ de amida III, respectivamente, e também em 1450 $\mathrm{cm}^{-1}$, atribuída à deformação do grupo $\mathrm{CH}_{2} \cdot{ }^{23}$ Carotenoides também poderiam ser detectados através de suas bandas características, em ca. $1150 \mathrm{~cm}^{-1} v(\mathrm{CC})$ e $1520 \mathrm{~cm}^{-1} v(\mathrm{C}=\mathrm{C}) .{ }^{15} \mathrm{~A}$ presença de $\alpha$-quartzo, geralmente detectado em $465 \mathrm{~cm}^{-1}$ (estiramento da ligação Si-O), poderia ser detectada nos espectros se areia tivesse sido incorporada como carga ou espessante. A literatura já reportou que o uso de água rica em $\mathrm{Ca}^{2+} \mathrm{e} \mathrm{CO}_{3}{ }^{2-}$, como é o caso da água do rio Peruaçu, pode assegurar uma boa aglutinação aos pigmentos após secagem, por meio de precipitação de calcita, ${ }^{7}$ uma vez que partículas de calcita podem aprisionar compostos como óxidos e hidróxidos de Fe e Mn, conferindo uma adesão duradoura entre as partículas dos pigmentos e entre estes e o suporte rochoso. Este ponto, entretanto, é de difícil verificação, uma vez que o próprio suporte é carbonático. Além disso, é possível também que substâncias orgânicas tenham sido eventualmente usadas, mas tenham se degradado ao longo do tempo.

\section{Produtos de degradações}

Em análises anteriormente reportadas, ${ }^{13}$ cortes estratigráficos de algumas amostras apresentavam fina camada de material branco sobre a pintura quando observados em estereoscópio, sendo que a análise por EDS indicou a presença de grande concentração de cálcio nessas áreas. De fato, em boa parte dos espectros Raman analisados foi observada a presença de outras bandas, além daquelas características dos pigmentos identificados. Em parte das amostras, chama a atenção o aparecimento de um dubleto em 1464 e $1492 \mathrm{~cm}^{-1}$. Essas bandas caracterizam as vibrações de estiramento dos grupos $-\mathrm{CO}_{2}$ do íon oxalato em wewelita $\left(\mathrm{CaC}_{2} \mathrm{O}_{4} \cdot \mathrm{H}_{2} \mathrm{O}\right) \cdot{ }^{15}$ Outras bandas que identificam esse composto situam-se em $895 \mathrm{~cm}^{1}$, atribuída à vibração de estiramento da ligação carbono-carbono, $v(\mathrm{C}-\mathrm{C})$, e em $502 \mathrm{~cm}^{-1}$, atribuída à deformação angular do grupo $-\mathrm{CO}_{2}$ (Figura $\left.5 \mathrm{~A}\right) .{ }^{15} \mathrm{~A}$ Figura $5 \mathrm{~A}$ 
também mostra que é possível identificar wewelita inequivocamente em amostras com diferentes pigmentações.

(A)
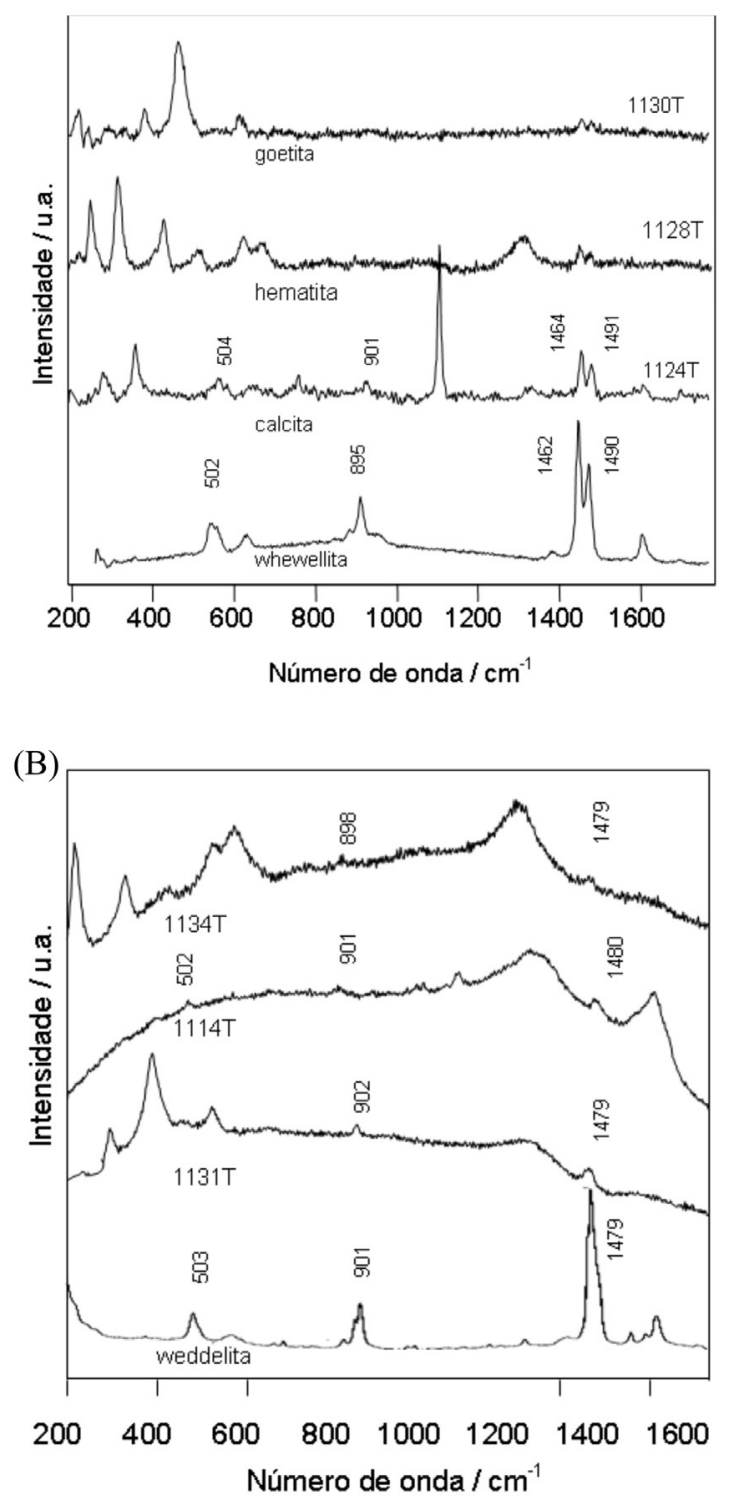

Figura 5. (A) Espectro de wewelita $\left(\mathrm{CaC}_{2} \mathrm{O}_{4} \cdot \mathrm{H}_{2} \mathrm{O}\right)$ presente como o par de bandas em 1462 e $1490 \mathrm{~cm}^{-1}$ em algumas das amostras de pigmento amarelo (1130T), pigmento vermelho (1128T) e calcita (1124T). (B) Espectro de wedelita $\left(\mathrm{CaC}_{2} \mathrm{O}_{4} \cdot 2 \mathrm{H}_{2} \mathrm{O}\right)$, cujas bandas principais em 901 e $1479 \mathrm{~cm}^{-1}$ estão presentes nos espectros de amostras dos pigmentos vermelho (1134T), preto (1114T) e amarelo (1131T)

Além da forma mono-hidratada, observou-se que amostras de pigmentos preto (1114T), vermelho (1134T) e amarelo (1131T) também apresentaram bandas características da forma di-hidratada de oxalato de cálcio (wedelita) em 504, $909 \mathrm{~cm}^{-1}$ e uma única banda em $1481 \mathrm{~cm}^{-1}$ que correspondem às vibrações $\delta\left(\mathrm{CO}_{2}\right), v(\mathrm{CC})$ e $v_{\text {sim }}\left(\mathrm{CO}_{2}\right)$, respectivamente (Figura 5B). ${ }^{15}$

Espectros FTIR reportados anteriormente para algumas amostras (1135T, 1134T, 1133T, 1130T, 1128T, 1121T, 1120T, 1114T, 1110T e $1101 \mathrm{~T})$ do Abrigo do Janelão apresentaram bandas características de oxalato de cálcio em $1640 \mathrm{~cm}^{-1}(\mathrm{v}(\mathrm{C}=\mathrm{O})), 1320 \mathrm{~cm}^{-1}(\mathrm{v}(\mathrm{C}-\mathrm{O}))$ e 780 $\mathrm{cm}^{-1}(\mathrm{v}(\mathrm{Ca}-\mathrm{O})+\delta(\mathrm{O}-\mathrm{C}-\mathrm{O})),{ }^{13}$ entretanto, essas bandas aparecem nas mesmas regiões nas quais os modos vibracionais do grupo carbonato também são observados, fazendo com que a identificação inequívoca de oxalato não seja uma tarefa simples. Além disso, a diferenciação do mono-hidrato e do di-hidrato por FTIR não é trivial, particularmente em amostras reais, que sabidamente têm composição complexa, devido à similaridade dos espectros, como mostrado na Figura 6 , com diferenciações pequenas entre um e outro. ${ }^{24}$

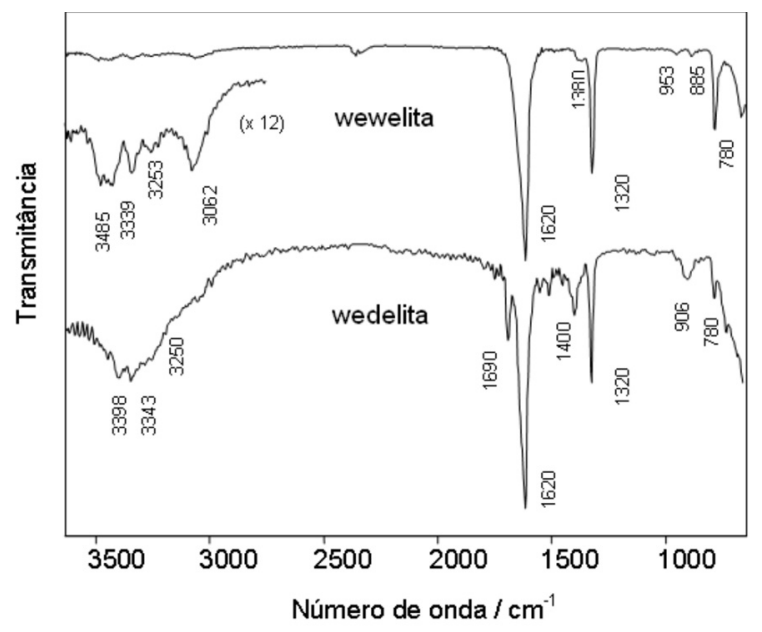

Figura 6. Espectros FTIR (ATR) de wewelita e wedelita

A comparação dos espectros mostrados na Figura 6 evidencia que, em frequências maiores (região de estiramento $\mathrm{O}-\mathrm{H}$ ), a wewelita apresenta uma banda em $3062 \mathrm{~cm}^{-1}$, ausente na wedelita. Sugeriu-se que essa região fosse usada na diferenciação dos espectros FTIR desses oxalatos, ${ }^{25}$ entretanto, em amostras reais é muito provável a superposição com bandas de outras substâncias, principalmente água e silicatos.

Por outro lado, os espectros Raman desses oxalatos apresentam claras diferenças na região entre 1460 e $1500 \mathrm{~cm}^{-1}$. Na wewelita, observa-se um par de bandas em 1464 e $1492 \mathrm{~cm}^{-1}$, enquanto que na wedelita, apenas uma banda única aparece em $1480 \mathrm{~cm}^{-1}$. Neste trabalho, a distinção dessas espécies foi feita com facilidade, em concordância com outros trabalhos da literatura. ${ }^{24}$

A presença de oxalato de cálcio em superfícies rochosas pode ser explicada tanto pela ocorrência natural quanto pela ação de microorganismos, como líquens e fungos, os quais excretam ácido oxálico como produto de seu metabolismo e que, ao contato com o substrato carbonático, produz o sal de cálcio. ${ }^{24} \mathrm{Em}$ algumas áreas do abrigo, foram observadas "incrustações brancas como início de infestação, cuja aparência esponjosa e circular parece evoluir radialmente, acarretando escamações e arraste de camadas de pintura". ${ }^{13}$ Nessas áreas, análise microbiológica realizada anteriormente ${ }^{13}$ identificou fungos dos gêneros Aespergillus, Penicillum e Cladosporium, que possuem atividade biocorrosiva e produzem, entre outros metabólitos, ácido oxálico. Como já mencionado, ácido oxálico secretado pelo microbionte causa erosão da rocha calcária e converte-se em oxalato de cálcio, que é acumulado na interface como uma incrustação entre o líquen e o substrato; altas concentrações de oxalato ocorrem também no talo do líquen. ${ }^{24}$

Sabe-se que os danos causados por líquens aparentemente provêm de uma combinação de efeitos físicos (fragmentação da rocha pela penetração da hifa ${ }^{26}$ e químicos (produção de ácidos e compostos polifenólicos, principalmente). ${ }^{27}$ Além disso, líquens criam seu próprio microclima e a produção de oxalato de cálcio mono e di-hidratado está relacionada à temperatura ambiente e umidade. Wadsten e Moberg observaram que líquens de locais úmidos produziam o mono-hidrato, 
enquanto aqueles que cresciam em locais mais secos produziam uma mistura de mono-hidrato e di-hidrato. ${ }^{28}$ Condições climáticas favoráveis podem, portanto, produzir a hidratação de oxalato de cálcio na interface líquen-substrato e isso explica porque wedelita, uma forma metaestável de oxalato de cálcio, é frequentemente associada a produtos de metabolismo de líquens. ${ }^{29}$

A detecção de oxalatos de cálcio hidratados em sítios arqueológicos pode ser uma potencial fonte de preocupação e de ação preventiva, pelo fato dessas substâncias estarem associadas à degradação microbiológica.

Outro aspecto interessante a ser abordado é a questão da cristalinidade dos pigmentos, a qual pode ser afetada pela presença de líquens, causando sua hidratação e posterior desordenamento da estrutura cristalina. A modificação de hematita já foi mencionada como estratégia de sobrevivência de cianobactérias em locais com condições ambientais altamente hostis, como a Antártica ${ }^{30}$ e a superfície de Marte. ${ }^{31}$ Há, entretanto, um grande interesse em definir se esse desordenamento pode decorrer da manipulação intencional de minérios visando a obtenção de novas tonalidades de cor. Esse ponto é abordado a seguir.

\section{Manipulação de pigmentos}

Especificamente no caso de óxidos de ferro, existe a possibilidade de modificação da cor amarela (goetita) para a vermelha (hematita) através do aquecimento em temperaturas relativamente baixas (menos de $400{ }^{\circ} \mathrm{C}$ ). A hematita preparada pela desidratação da goetita nessas condições apresenta estrutura amorfa, ${ }^{32-35}$ causada pelo arranjo imperfeito dos átomos de ferro e oxigênio, a qual se reflete no espectro Raman como alargamento de bandas e aumento na intensidade da banda em ca. $660 \mathrm{~cm}^{-1}$. O mesmo efeito é observado também nos difratogramas de raios $\mathrm{X}$, frequentemente reportados em estudos desse tipo. Caso o aquecimento fosse a única origem provável para a ocorrência dessa diminuição de cristalinidade, tanto a espectroscopia Raman quanto a difração de raios X poderiam ser usadas na identificação de manipulação de pigmentos, indicando conhecimento técnico por parte daquela comunidade. Ocorre que intemperismos podem também causar as mesmas modificações estruturais, como é visto no caso da limonita (mistura de goetita e hematita amorfa). ${ }^{36}$ Espectros obtidos de amostras coletadas do entorno do sítio arqueológico (Figura 7) apresentaram bandas alargadas, dando suporte a essa suposição.

Uma característica interessante dos espectros de hematita amorfa ou proveniente de goetita processada termicamente é a presença de uma banda relativamente intensa e larga em ca. $660 \mathrm{~cm}^{-1}$, que tanto pode indicar a presença de hematita amorfa quanto de magnetita $\left(\mathrm{Fe}_{3} \mathrm{O}_{4}\right)$. $\mathrm{O}$ espectro Raman da magnetita apresenta como banda mais intensa aquela relativa à vibração de $\mathrm{Fe}-\mathrm{O}$ com coordenação tetraédrica, que aparece em $667 \mathrm{~cm}^{-122}$ e sabe-se que é possível obter hematita a partir de magnetita através de um processo conhecido como martitização, que pode ser levado a cabo tanto térmica quanto mecanicamente ${ }^{37}$ Se a transformação não é completa é possível detectar as duas substâncias, assim, o aquecimento de magnetita (pigmento preto) poderia levar à formação de hematita (pigmento vermelho) e a presença da banda larga e intensa em $660 \mathrm{~cm}^{-1}$ poderia ser um argumento a favor dessa suposição. Essa hipótese, entretanto, não sobrevive a uma comparação mais cuidadosa dos espectros, como já mostrado anteriormente..$^{12}$

\section{CONCLUSÕES}

Neste trabalho estão reportados os resultados referentes a 14 amostras coletadas de pinturas rupestres (Abrigo Norte do Janelão, Parque Nacional Cavernas do Peruaçu), visando a identificação das

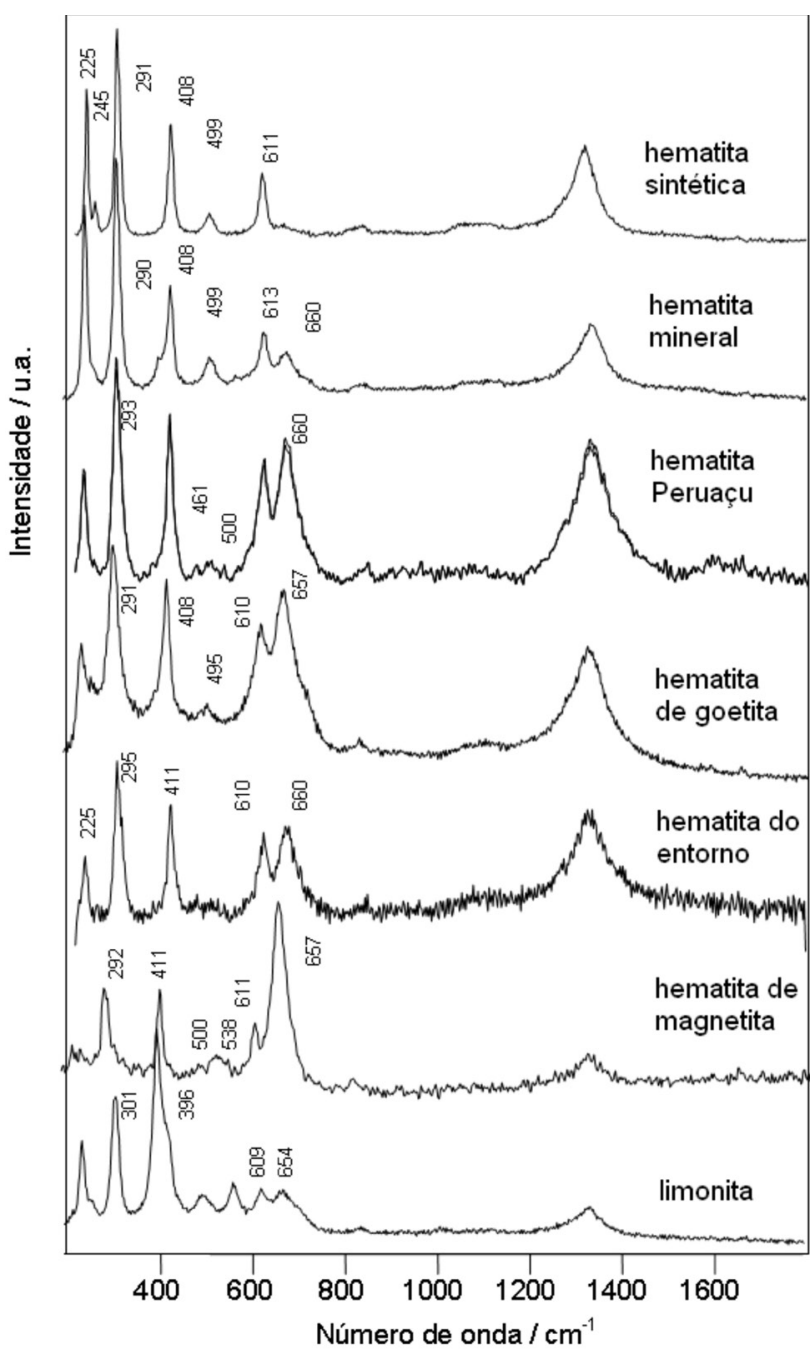

Figura 7. Espectros Raman $(632,8 \mathrm{~nm})$ de hematita de diversas procedências

substâncias presentes, sua origem e eventuais interações e produtos de degradação.

Não foram detectados possíveis aglutinantes orgânicos (gordura ou cola animal, sangue, albumina etc.) e produtos de degradação foram identificados predominantemente como oxalatos de cálcio, nas formas mono-hidratada (wewelita) e di-hidratada (wedelita), que aparecem como resultado da reação de ácido oxálico (produto do metabolismo de micro-organismos, como líquens) e o substrato carbonático. A resolução espacial inerente à microscopia Raman possibilitou a identificação dessas duas espécies em diferentes amostras; a distinção entre essas duas formas em amostras reais através de FTIR não é tão simples e muitas vezes é impossibilitada pela composição da amostra estudada.

No que diz respeito à manipulação ou não de pigmentos pelos paleoíndios, as amostras de pigmentos vermelhos identificadas no Vale do Peruaçu apresentam padrão de hematita desordenada, que se assemelham em termos de intensidades relativas à goetita aquecida entre $250-600{ }^{\circ} \mathrm{C}$, entretanto, outros fatores como intemperismos, por exemplo, levam às mesmas alterações e, de fato, amostras coletadas no entorno do sítio arqueológico apresentaram o mesmo comportamento.

\section{REFERÊNCIAS}

1. Pessis, A. M.; Imagens da Pré-História - Parque Nacional Serra da Capivara, FUMDHAM/Petrobrás: Rio de Janeiro, 2003. 
2. Pereira, E.; Arte rupestre na Amazônia - Pará, Ed. Unesp: Araraquara, 2004; Gaspar, M. D.; Arte Rupestre no Brasil, Ed. Jorge Zahar: Rio de Janeiro, 2003; Lopes, F. N.; Dissertação de Mestrado, Universidade de São Paulo, Brasil, 2007.

3. http://www.fumdham.org.br/publicacoes.html, acessada em Julho 2010.

4. Prous, A.; Arqueologia Brasileira, Ed. da UnB: Brasília, 2003.

5. Afonso, M. C.; Piedade, S. C. M.; Morais, J. L.; Revista do Museu de Arqueologia e Etnologia 1999, 9, 23.

6. Faria, D. L. A.; Afonso, M. C.; Edwards, H.G.M.; Revista do Museu de Arqueologia e Etnologia 2003, 12, 249.

7. Smith, D. C.; Bouchard, M.; Lorblanchet, M.; J. Raman Spectrosc. 1999, 30, 347 .

8. Vandenabeele, P.; Wehling, B.; Moens, L.; Edwards, H. G. M.; De Reu, M.; van Hooydonk, G.; Anal. Chim. Acta 2000, 407, 261.

9. Russ, J.; Kaluarachi, W. D.; Drummond, L.; Edwards, H. G. M.; Studies in Conservation 1999, 44, 91.

10. Hernanz, A.; Mas, M.; Gavilán, B.; Hernández, B.; J. Raman Spectrosc. 2006, 37, 492

11. Maier, M. S.; de Faria, D. L. A.; Boschin, M. T.; Parera, S.D.; del Castillo Bernal, M. F.; Vib. Spectrosc. 2007, 44,182.

12. de Faria, D. L. A.; Lopes, F. N.; Vib. Spectrosc. 2007, 45, 117.

13. Branco, H. D. O. C.; Dissertação de Mestrado, Universidade Federal de Minas Gerais, Brasil, 2001.

14. Wiedemann, G.; Bayer, G.; J. Thermal. Anal. 1988, 33, 707.

15. Edwards, H. G. M.; Farwell, D. W.; Jenkins, R.; Seaward, M. R. D.; J. Raman Spectrosc. 1992, 23, 185.

16. Ferrari, A. C.; Robertson, J.; Phys. Rev. B 2000, 61, 14095.

17. Buciuman, F.; Patcas, F.; Craciun, R.; Zhan, D. R. T.; Phys. Chem. Chem. Phys. 1999, 1, 185.

18. Martin, T. P.; Merlin, R.; Huffman, D. R.; Cardona, M.; Solid State Commun.1977, 22, 565.
19. McCarty, K. F.; Solid State Commun. 1988, 68, 799.

20. Kontoyannis, C. G.; Vagenas, N. V.; Analist 2000, 125, 251.

21. Frost, R. L.; Fredericks, P. M.; Spectrochim. Acta Part A 1993, 49, 667; Michaelian, K. H.; Can. J. Chem. 1986, 64, 285.

22. Faria, D. L. A.; Silva, S. V.; de Oliveira, M. T.; J. Raman Spectrosc. 1997, 28, 873.

23. Pelton, J. T.; McClean, L. R.; Anal. Biochem. 2000, 277, 167.

24. Edwards, H. G. M.; Farwell, D. W.; Daffner, L.; Spectrochim. Acta Part A 1996, 52, 1639; Edwards, H. G. M.; Edwards, K. A. E.; Farwell, D. W.; Lewis, I. R.; Seaward, M. R. D.; J. Raman Spectrosc. 1994, 25, 99.

25. Frost, R. L.; Anal. Chim. Acta 2004, 517, 207.

26. Fry, E. J.; Ann. Bot. (London) 1924, 38, 175.

27. Syers, J. K.; Iskandar, I. K.; The Lichenologist 1971, 5, 45; European J. Soil Sci. 1972, 23, 255.

28. Wadsten, T.; Moberg, R.; The Lichenologist 1985, 17, 239.

29. Edwards, H. G. M.; Russell, N. C.; Seaward, M. R. D.; Slarke, D.; Spectrochim. Acta Part A 1995, 51, 2091.

30. Edwards, H. G. M.; Wynn-Williams, D. D.; Villar, S. E. J.; J. Raman Spectrosc. 2004, 35, 470.

31. Edwards, H. G. M.; Newton, E. M. Em The Search for Life on Mars; Hiscox, J., ed.; British Interplanetary Society: London, 1999, p. 83.

32. Yariv, S.; Mendelovici, E.; Villalba, R.; J.Chem. Soc. Faraday I 1980, 76,1442 .

33. Francombe, M.; Rooksby, H .G.; Clay Mineral. Bull. 1959, 4, 1.

34. Lima-de-Faria, J.; Z. Krist. 1963, 119, 176.

35. Brown, G. Em Crystal Structures of Clay Minerals and Their X-Ray Identification; Brindley, G.; Brown, G., eds.; Mineral. Soc.: London, 1984, p. 373.

36. Holser, W. T.; Acta Crystal. 1953, 6, 565.

37. Ramdohr, P.; The Ore Minerals and Their Intergrowths, Pergamon Press: Oxford, 1969, p. 980. 